\title{
The conjugacy problem in subgroups of right-angled Artin groups
}

\author{
John Crisp, Eddy Godelle, and Bert Wiest
}

\begin{abstract}
We prove that the conjugacy problem in a large and natural class of subgroups of right-angled Artin groups (RAAGs), can be solved in linear-time. This class of subgroups has been previously studied by Crisp and Wiest, and independently by Haglund and Wise, as fundamental groups of compact special cube complexes.
\end{abstract}

\section{Introduction}

It is well known that the conjugacy problem in free groups can be solved in linear-time by a RAM (random access memory) machine. This result has been generalized in two different directions. On the one hand, Epstein and Holt [14] have shown that the conjugacy problem is linear in all word-hyperbolic groups. On the other hand, Liu, Wrathall and Zeger have proved the analogue result for all right-angled Artin groups ([24], based on [31]). Note that the latter groups are also called "partially commutative groups" or "graph groups" in the literature.

The aim of the present paper is to further generalize the second result, by proving linearity of the conjugacy problem in a large class of subgroups of right-angled Artin groups. This class of groups has previously been studied by Crisp and Wiest $[\mathbf{1 1}, \mathbf{1 2}]$, and independently by Haglund and Wise [19]; these groups are fundamental groups of certain cube complexes, called compact $A$-special cube complexes in [19].

Our generalization is based on a new solution to the conjugacy problem in right-angled Artin groups (RAAGs), different from the one of Liu, Wrathall and Zeger, but rather close in spirit to the methods of Lalonde and Viennot $[\mathbf{2 3}, \mathbf{3 0}]$.

The class of groups considered in this paper contains in particular all graph braid groups [1, $2,15,16,26]$ and more generally all state complex groups $[3,17]$, which are closely related to robotics $[\mathbf{2}, \mathbf{1 7}]$ - indeed, our results can be interpreted as giving very efficient algorithms for motion planning of periodic robot movements. Notice that it is still unknown whether Coxeterand Artin groups are virtually fundamental groups of compact special cube complexes $[\mathbf{1 9}, \mathbf{2 0}$, so our results do not apply immediately to these groups.

The plan of the paper is as follows. In the second section we present our new solution to the conjugacy problem in RAAGs. In the third section we give a precise definition of the class of subgroups of RAAGs under consideration, and prove that they inherit a linear-time solution to the conjugacy problem from their supergroups.

\section{The conjugacy problem in RAAGs is linear-time}

We recall that a right-angled Artin group is a group given by a finite presentation, where every relation states that some pair of generators commutes. Graphically, a right-angled Artin group $A$ can be specified by a simple graph $\Gamma_{A}$, where the generators of $A$ correspond to the vertices of $\Gamma_{A}$, and a pair of generators commutes if and only if the corresponding vertices are 
not connected by an edge. Note that the opposite convention (connecting commuting generators by an edge) is also very common, but in the present paper we shall stick to this convention.

Right-angled Artin groups have been widely studied in the last decades - see [10] for an excellent survey. Several solutions to the word and conjugacy problem have been found. It seems difficult to have a complete bibliography concering these two problems, but the articles by Servatius [27], Van Wyk [29], Liu, Wrathall and Zeger [24] (based on [31]), Cartier and Foata [9], Viennot [30], Lalonde [23], and Krob, Mairesse, and Michos [22] can be highlighted.

In order to approach the conjugacy problem in right-angled Artin groups, let us first consider the relatively easy case of free groups. Given two cyclic words of length $\ell_{1}$ and $\ell_{2}$ respectively, there is a two step algorithm which can be performed in time $O\left(\ell_{1}+\ell_{2}\right)$ on a RAM machine: first each word can be cyclically reduced in time $O\left(\ell_{1}\right)$ and $O\left(\ell_{2}\right)$, respectively. If the reduced words have different lengths, then they are not conjugate. If they have the same length $\ell$, then they can be compared in time $O(\ell)$ using standard pattern matching algorithms, like the Knuth-Morris-Pratt algorithm, the Boyer-Moore algorithm, or algorithms based on suffixtree methods - see $[\mathbf{2 1}, \mathbf{7}, \mathbf{4}, \mathbf{1 8}, \mathbf{2 8}]$. It should be stressed that on a Turing machine these algorithms take time $O(\ell \log (\ell))$.

In the sequel, we assume that $A$ is a fixed right-angled Artin group given by a fixed presentation. We denote by $\left\{a_{1}, \cdots, a_{N}\right\}$ the generating set of $A$ associated with this presentation.

In this section we shall provide an algorithm for the conjugacy problem in $A$ which works as follows: given a word $w$, another word $w^{\prime}$ with smaller or equal length is created in linear time such that $w$ and $w^{\prime}$ represent conjugate elements of $A$. Furthermore, the word $w^{\prime}$ depends only on the conjugacy class in $A$ of the element represented by $w$, up to a cyclic permutation of its letters. This yields a linear-time solution to the conjugacy problem in $A$ because, given words $w$ and $v$ we can compute the canonical cyclic words $w^{\prime}$ and $v^{\prime}$ representing their conjugacy classes, and compare those by one of the algorithms mentioned above.

\subsection{Normal forms and pilings}

In this subsection we reprove the well-known fact that there is a linear-time solution to the word problem. We start by recalling the following classical lemma.

Lemma 2.1. [27] Any element of $A$ can be represented by a reduced word (one which does not contain a subword of the form $a_{i}^{ \pm 1} x a_{i}^{\mp 1}$, where all letters of $x$ commute with $a_{i}$ ). Moreover, any two reduced representatives of the same element are related by a finite number of commutation relations - no insertions/deletions of trivial pairs are needed.

Now we introduce our main tool, the notion of a piling.

Definition 2.2. An abstract piling is a collection of $N$ words, one for each generator $a_{i}$ of $A$, over the alphabet with three symbols $\{+,-, 0\}$.

The word associated with the generator $a_{i}$ will be called the $a_{i}$-stack of the abstract piling. The product of two abstract pilings is defined as the piling obtained by concatenation of the corresponding stacks.

We define a function $\pi^{\star}$ that associates to every word on the $2 n$ letters $a_{1}, a_{1}^{-1}, \ldots, a_{N}, a_{N}^{-1}$ an abstract piling in the following way: starting with the empty piling, we read the word from left to right. When a letter $a_{i}^{\epsilon}$ is read, we check what the last letter of the $a_{i}$-stack of the piling 
is. If this letter is different from $-\epsilon$ (the no-cancellation cases) then we append a letter + or (the sign of $\epsilon$ ) at the end of the $a_{i}$-stack of the piling. Moreover, we also append a letter 0 at the end of each of the $a_{j}$-stacks associated with a generator $a_{j}$ that does not commute with the generator $a_{i}$. On the other hand, if the last letter of the $a_{i}$-stack is $-\epsilon$ (the cancellation case), then we erase this last letter, and we also erase the terminal letter of each of the $a_{j}$-stacks of the piling associated with a generator $a_{j}$ that does not commute with the generator $a_{i}-$ note that the terminal letter of the $a_{j}$-stack is necessarily " 0 ".

Definition 2.3. A piling is an abstract piling in the image of the function $\pi^{\star}$. The set of pilings is denoted $\Pi$.

We observe that the number of letters + and - occuring in the piling $\pi^{\star}(w)$ is at most equal to the length of the word $w$. Moreover, it is immediate from the description of the function $\pi^{\star}$ that, given a word $w$ of length $\ell$, the piling $\pi^{\star}(w)$ can be calculated in time $O(\ell)$ (linear-time).

It may be helpful to keep in mind the following physical interpretation of a piling: we have $N$ vertical sticks, labelled by the generators $a_{1}, \ldots, a_{n}$, with beads on it; the beads are labelled by,+- or 0 such that when reading from bottom to top the sequence of labels of the beads on the $a_{i}$-stick, we obtain the $a_{i}$-stack of the piling. A letter $a_{i}$ or $a_{i}^{-1}$ of the word $w$ corresponds to a set of beads (which we call a tile), consisting of one bead labelled + or - on the corresponding stick, and one bead labelled 0 on each of the sticks corresponding to generators of $A$ which do not commute with $a_{i}$; each 0 labelled bead is connected to the \pm labelled bead by a thread. On a stick, adjacent 0-beads can commute with ("slide through") each other, but 0-beads do not commute with \pm -beads.

EXAMPLE 2.4. In the group $A$ with group presentation

$$
\left\langle a_{1}, a_{2}, a_{3}, a_{4} \mid a_{1} a_{4}=a_{4} a_{1} ; a_{2} a_{3}=a_{3} a_{2} ; a_{2} a_{4}=a_{4} a_{2}\right\rangle
$$

we can calculate the piling $p$ of the word $a_{2}^{-2} a_{4}^{-1} a_{3} a_{2} a_{4} a_{1} a_{2} a_{1}^{-1} a_{2}^{2} a_{4}^{-1}$ as indicated in Figure 1 .
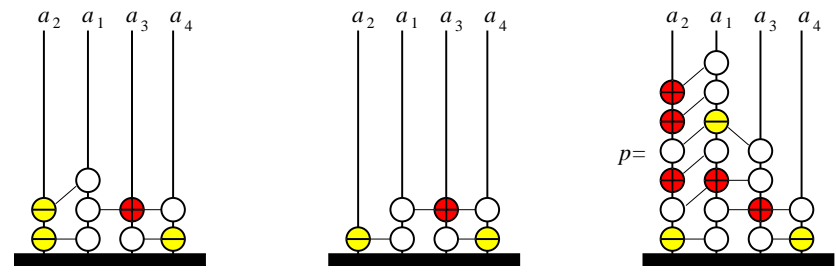

Figure 1. The pilings of the prefixes $a_{2}^{-2} a_{4}^{-1} a_{3}$ and $a_{2}^{-2} a_{4}^{-1} a_{3} a_{2}$, and of the full word $a_{2}^{-2} a_{4}^{-1} a_{3} a_{2} a_{4} a_{1} a_{2} a_{1}^{-1} a_{2}^{2} a_{4}^{-1}$

The map $\pi^{\star}$ induces a well-defined function $\pi: A \rightarrow \Pi$ because words representing the same element of $A$ have the same image under $\pi^{\star}$ : the image of a word is unchanged by applying a commutation relation, and by inserting or deleting a trivial pair $a_{i} a_{i}^{-1}$ or $a_{i}^{-1} a_{i}$. Now, from the definitions it is immediate that no cancellation occurs during the construction of the piling $\pi^{\star}(w)$ of a reduced word $w$. Thus the identity of $A$ is the unique element of $A$ whose image by $\pi$ is the trivial piling, and therefore the word problem is solved in linear-time: a word $w$ represents the identity if and only if its piling $\pi^{\star}(w)$ is trivial; this piling can be built in linear-time. 
Definition 2.5. Let $w$ be a reduced word belonging to $\left\{a_{1}^{ \pm 1}, \ldots, a_{N}^{ \pm 1}\right\}^{*}$.

(i) We say that $w$ is initially normal when $w$ is trivial or when the index of its first letter is greater or equal to the index of the first letter of any equivalent reduced word.

(ii) We say that $w$ is normal when all its suffixes are initially normal.

We remark that all the factors of a normal word are normal words.

Proposition 2.6. Any element of $A$ has a unique normal reduced representative word.

Proof. For any reduced word $w=a_{i_{1}}^{\varepsilon_{1}} \cdots a_{i_{k}}^{\varepsilon_{k}}$, where $\varepsilon_{j}= \pm 1$, we set

$$
\Omega(w)=\left\{(r, s) \mid 1 \leq r<s \leq k \text { and } i_{r}<i_{s}\right\} .
$$

Let $a$ be in $A$. In order to prove that $a$ has normal reduced representative word, we choose, among all words representing $a$, a word $w$ for which the number $\# \Omega(w)$ is as small as possible (possibly equal to zero). This word $w$ is minimal.

We shall prove uniqueness of the normal representative by induction on the length. If $a$ is of length 1, i.e. if $a=a_{i}^{\varepsilon}$ for $\epsilon= \pm 1$, then uniqueness is obvious. Now suppose that $a$ has two normal reduced representatives $w=a_{i_{1}}^{\varepsilon_{1}} \cdots a_{i_{k}}^{\varepsilon_{k}}$ and $w^{\prime}=a_{i_{1}^{\prime}}^{\varepsilon_{1}^{\prime}} \cdots a_{i_{k}^{\prime}}^{\varepsilon_{k}^{\prime}}$. Since the the suffixes of length $k-1$ of $w$ and $w^{\prime}$ are again normal, it is, by induction hypothesis, sufficient to prove that $a_{i_{1}}^{\varepsilon_{1}}=a_{i_{1}^{\prime}}^{\varepsilon_{1}^{\prime}}$. Since $w$ and $w^{\prime}$ are normal, we have $i_{1}=i_{1}^{\prime}$. Now, the exponents also have to be equal by Lemma 2.1: we can not transform the word $a_{i_{1}}^{\varepsilon_{1}} \cdots a_{i_{k}}^{\varepsilon_{k}}$ into the word $a_{i_{1}}^{-\varepsilon_{1}} a_{i_{2}^{\prime}}^{\varepsilon_{2}^{\prime}} \cdots a_{i_{k}^{\prime}}^{\varepsilon_{k}^{\prime}}$ by using commutation relations only: starting from the reduced $w$, no word of the form $u a_{i_{1}}^{\varepsilon_{1}} a_{i_{1}}^{-\varepsilon_{1}} u^{\prime}$ can appear by any sequence of commutation relations.

In the sequel, we call this unique normal reduced word representing $a$ the normal form of $a$.

Proposition 2.7. There is a linear-time algorithm that associates to each piling $p$ a normal word $\sigma^{\star}(p)$ such that $\pi^{\star}\left(\sigma^{\star}(p)\right)=p$. Furthermore, for any element $a$ of $A$ the word $\sigma^{\star}(\pi(a))$ is the normal form of $a$.

Proof. Let $p$ be a piling. By definition, this means that there exists an element $a$ of $A$ such that $\pi(a)=p$. In order to prove Proposition 2.7, it suffices to find an algorithm for constructing in linear time a word $\sigma^{\star}(p)$, and to prove that $\sigma^{\star}(p)$ is a normal reduced representative of $a$.

We start with the observation that the element $a$ has a reduced representative starting with the letter $a_{i}^{ \pm 1}$ if and only if the $a_{i}$-stack of the piling is nonempty and starts with the letter + or - , respectively (not with the letter 0 ).

We associate to $p$ a normal reduced word $\sigma^{\star}(p)$ by induction on the number of letters + and - in $p$ in the following way. If $p$ is empty then $\sigma^{\star}(p)$ is the empty word. Otherwise, let $i$ be the largest index with the property that the $a_{i}$-stack of $p$ is nonempty and starts with the letter + or - . Then, according to this sign, we define the first letter of $\sigma^{\star}(p)$ to be $a_{i}$ or $a_{i}^{-1}$, respectively. Then we remove the tile consisting of the first letter $\left(+\right.$ or - ) of the $a_{i}$-stack, and of the initial letter (which has to be 0 ) of each of the $a_{j}$-stacks associated with a generator $a_{j}$ that does not commute with $a_{i}$. What remains is a piling $p_{1}$ with strictly fewer letters. Thus the word $\sigma^{\star}\left(p_{1}\right)$ is already defined, by induction hypothesis, and we define the word $\sigma^{\star}(p)$ by concatenation $\sigma^{\star}(p)=a_{i}^{ \pm 1} \sigma^{\star}\left(p_{1}\right)$.

We claim that the word $\sigma^{\star}(p)$ is a normal reduced representative of $a$; indeed, in the above construction we see that the first letter of $\sigma^{\star}(p)$ is also the first letter of some reduced 
representative of $a$. By induction, the whole word $\sigma^{\star}(p)$ is a reduced representative of $a$. Moreover, the word $\sigma^{\star}(p)$ is initially normal, by construction, and by induction its suffix $\sigma^{\star}\left(p_{1}\right)$ is normal. Hence the whole word $\sigma^{\star}(p)$ is normal.

EXAMPLE 2.8. Using the notation of Example 2.4, the word $\sigma^{\star}(p)$ is equal to $a_{4}^{-1} a_{3}$ $a_{2}^{-1} a_{1} a_{2} a_{1}^{-1} a_{2} a_{2}$. The calculation is shown in Figure 2 .

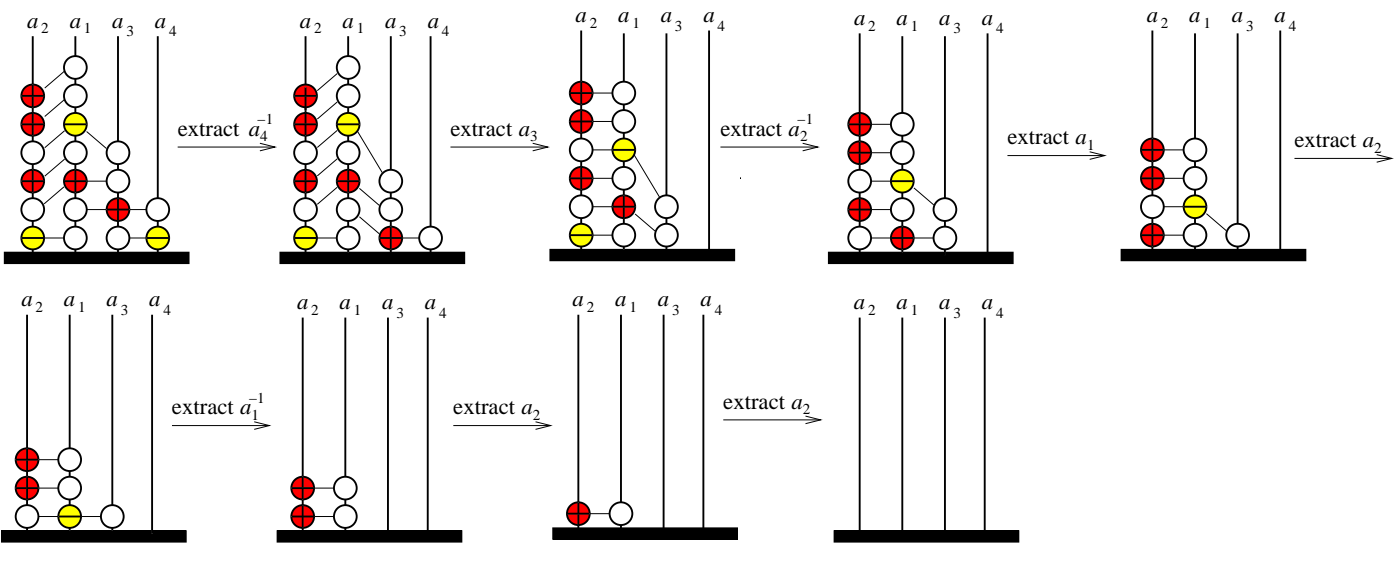

Figure 2. The word $\sigma^{\star}(p)$ associated to a piling $p$

\subsection{Cyclic normal forms and pyramidal pilings}

We are now ready to attack the conjugacy problem.

2.2.1. Cyclically reduced words and cyclically reduced pilings. We recall that a cycling of a reduced word $w$ is the operation of removing the first letter of the word, and placing it at the end of the word. A word is called cyclically reduced if it is reduced and if any word obtained from it by a sequence of cyclings and commutations is still reduced - in other words, if it is not of the form $x_{1} a_{i}^{ \pm 1} x_{2} a_{i}^{\mp 1} x_{3}$, where all the letters of $x_{1}$ and $x_{3}$ commute with $a_{i}$. As far as we know, all known solutions to the conjugacy problem in RAAGs are based on the following lemma.

Lemma 2.9. Two cyclically reduced words represent conjugate elements if and only if they are related by a sequence of cyclings and commutation relations.

Therefore two reduced words $w_{1}, w_{2}$ with letters in $\left\{a_{1}^{ \pm 1}, \ldots, a_{n}^{ \pm 1}\right\}$ represent conjugate elements of $A$ if and only if there is a sequence of words

$$
w_{1} \stackrel{\text { red }}{\longrightarrow} v_{1} \leftrightarrow v_{2} \stackrel{\text { red }}{\longleftarrow} w_{2}
$$

where the two arrows labelled "red" represent two sequences of cyclic reductions down to cyclically reduced words and the arrow $\leftrightarrow$ represents a finite sequence of cyclings and commutation relations. 
DEFINITION 2.10. If, in a piling $p$, the $a_{i}$-stack starts (resp. finishes) with a letter + or - , the bottom $a_{i}$-tile (resp. the top $a_{i}$-tile) of $p$ is the sub-piling formed by the first (resp. last) letter of the $a_{i}$-stack and the first (resp. last) letter of the $a_{j}$-stacks such that $a_{i}$ and $a_{j}$ do not commute in $A$.

ExAmple 2.11. With the notation of Example 2.4, Figure 3 gives an example of top and bottom tiles of a piling.

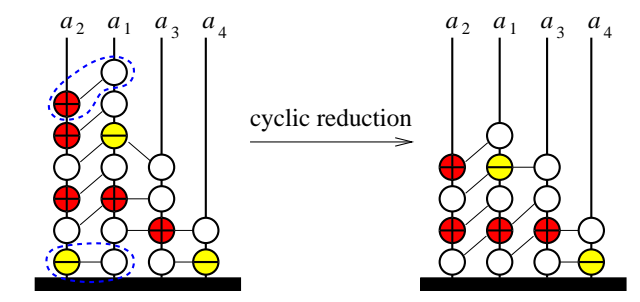

Figure 3. A top $a_{2}$-tile and a bottom $a_{2}$-tile, and the associated cyclic reduction

DeFinition 2.12. If in a piling $p$ the $a_{i}$-stack starts with the letter + and ends with - , or vice versa, a cyclic reduction is the act of removing both top and bottom $a_{i}$-tiles. We say that the piling is cyclically reduced if no cyclic reduction is possible.

Note that cyclically reducing a piling yields again a piling. We remark that there is an obvious linear-time algorithm for transforming any piling into a cyclically reduced one by a finite sequence of cyclic reductions. We also observe that for a reduced word $w \in\left\{a_{1}^{ \pm 1}, \ldots, a_{N}^{ \pm 1}\right\}^{*}$, cycling of $w$ corresponds to a cycling of its piling, and that $w$ is cyclically reduced if and only if the piling $\pi^{\star}(w)$ is.

Now we have a fast algorithm for cyclically reducing words and pilings. In contrast to the case of free groups, however, the reduced words which can obtained are not unique up to cyclic permutation. In order to circumvent this problem, we shall introduce in the sequel the notion of a cyclic normal form.

2.2.2. Non-split words and non-split pilings. Our first objective is to restrict the conjugacy problem to the case of non-split cyclically reduced words (or pilings). We recall that a graph $\Gamma_{A}$ is associated to the right-angled Artin group $A$.

Definition 2.13. Let $w$ be a reduced word different from 1 , and let $p$ be its image by $\pi^{\star}$. Consider $\Delta(p)$ (or $\Delta(w))$ the full subgraph of $\Gamma_{A}$ whose vertices are those whose correponding stacks contain at least one bead different from 0 (in other words, the letters $a_{i}$ such that $a_{i}^{ \pm 1}$ occurs in $w)$. Then, the word $w$ and the piling $p$ are said to be non-split when the graph $\Delta(p)$ is connected.

In other words, $w$ is non-split if and only if its set of letters cannot be separated in two disjoint subsets such that every letter of one of the subset commutes in $A$ with every letter of the other subset. Clearly, it takes linear-time to obtain the set of vertices of the graph $\Delta(p)$, and constant time (which depends on the graph $\Gamma_{A}$ ) to decide whether $\Delta(p)$ is connected. If it is not, it takes still constant time to determine the connected components $\Delta_{1}(w), \ldots, \Delta_{k}(w)$ 
of $\Delta(w)$. Figure 4 (which still uses the notation of Example 2.4) contains examples of both split and non-split pilings.

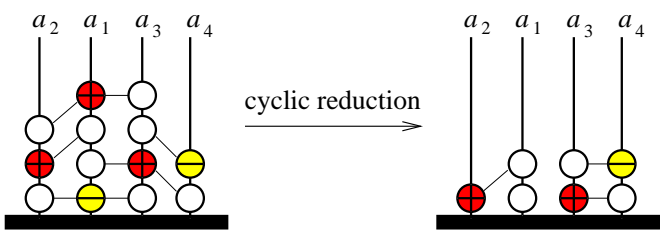

FIGURE 4. The word $a_{1}^{-1} a_{2} a_{3} a_{1} a_{4}^{-1}$ is not split, but cyclic reduction yields a word which is split: $a_{2}\left(a_{3} a_{4}^{-1}\right)=\left(a_{3} a_{4}^{-1}\right) a_{2}$

Now, if $w$ is a cyclically reduced word that is split, then it is equivalent to a product $w_{1} \cdots w_{k}$ of non-split cyclically reduced words, one for each connected component $\Delta_{i}(w)$ of the graph $\Delta(w)$; the graph $\Delta_{i}(w)$ is equal to $\Delta\left(w_{i}\right)$. Furthermore, once that the connected components $\Delta_{1}(w), \ldots, \Delta_{k}(w)$ of $\Delta(w)$ are computed, appropriate words $w_{1}, \ldots, w_{k}$ can be obtained in linear-time.

REMARK 2.14. The following observation will be crucial: if $v$ is another cyclically reduced word, then $w$ and $v$ represent conjugate elements if and only if two conditions are satisfied: firstly the graph $\Delta(v)$ is equal to $\Delta(w)$; secondly, if $v_{1}, \ldots, v_{k}$ are words such that $\Delta\left(v_{i}\right)=\Delta_{i}(v)$ and such that $v$ is equivalent to the product $v_{1} \cdots v_{k}$, then for each index $i$ the words $w_{i}$ and $v_{i}$ represent conjugate elements.

Therefore, in order to obtain a solution to the conjugacy problem in linear-time it is enough to consider the case of cyclically reduced non-split words.

2.2.3. Pyramidal piling and cyclic normal form To solve the conjugacy problem, we associate in the sequel a cyclic normal word to each cyclically reduced non-split word. We first do the analogue of this in the framework of pilings: to each non-split cyclically reduced piling, we associate a pyramidal piling.

Definition 2.15. Let $p$ be a non-empty piling, and denote by $i$ the smallest index such that the $a_{i}$-stack contains an $a_{i}^{ \pm}$-bead. We say that the piling $p$ is pyramidal if the first bead of every stack except the $a_{i}$-stack is either empty or starts with the letter 0 . In that case, we say that $a_{i}$ is the apex of the pyramidal piling.

Note that a pyramidal piling has to be non-split.

LEMma 2.16. (i) Let $p$ be a non-empty piling and denote by $i$ the smallest index such that the $a_{i}$-stack of $p$ contains an $a_{i}^{ \pm}$-bead; then there exists a unique decomposition $p_{0} \cdot p_{1}$ of $p$ such that $p_{1}$ is a pyramidal piling with $a_{i}$ as apex, and $p_{0}$ is a piling without $a_{i}^{ \pm}$-beads. Furthermore, one has the equality of words $\sigma^{\star}(p)=\sigma^{\star}\left(p_{0}\right) \sigma^{\star}\left(p_{1}\right)$.

(ii) The above decomposition $p_{0} \cdot p_{1}$ can be computed in linear-time on the number of \pm beads of the piling $p$.

ExAmple 2.17. Using the notation of Example 2.4, Figure 5 gives an example of a decomposition of a piling. 


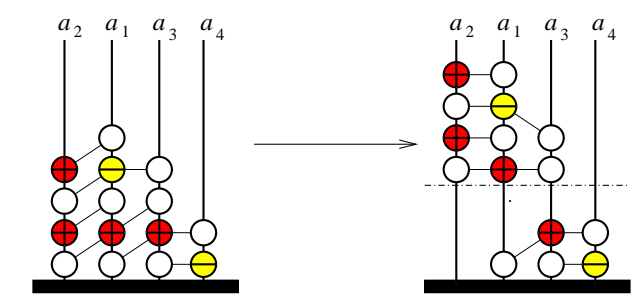

Figure 5. Decomposition of a piling as $p_{0} \cdot p_{1}$

Proof of Lemma 2.16. We start by exhibiting a linear-time algorithm for finding such a decomposition of a given non-empty piling $p$. Let $p_{0}$ be the empty piling. Reading all the stacks (in the index order), obtain in linear-time the smallest index $i$ for which the $a_{i}$-stack contains a bead distinct from 0 . Then, apply iteratively the following recipe: consider the largest index $j$ (necessarily greater than $i$ ) for which the $a_{j}$-stack starts with a letter + or - ; then remove all the beads in the bottom $a_{j}$-tile, and add them to the top of the piling $p_{0}$. When no more beads can be extracted from the bottom of the piling $p$, then the construction of the factor $p_{0}$ is complete, and what remains is the piling $p_{1}$. This proves the existence part of (i), as well as part $(i i)$. The formula $\sigma^{\star}(p)=\sigma^{\star}\left(p_{0}\right) \sigma^{\star}\left(p_{1}\right)$ is now immediate by construction. For the uniqueness part of (i), we notice that in any decomposition $p=p_{0} \cdot p_{1}$, the factor $p_{0}$ has to contain exactly those tiles that can be extracted on the bottom from $p$ without extracting any apex bead.

We call the piling $p_{0}$ the 0 -factor of $p$. Thus the piling $p$ is pyramidal if and only if its 0 -factor is empty.

In our physical interpretation, if $i$ is the smallest index such that the $a_{i}$-stack contains a $a_{i}^{ \pm}$bead, we can lift up the first $a_{i}^{ \pm}$-bead along its stick to the first floor. Then some part of the piling stays on the ground, while some beads are lifted up. The factor that stays down is $p_{0}$, the factor that is lifted up is $p_{1}$. This latter factor has the structure of an upside-down pyramid supported by one of the apex-beads, hence the names.

If in a cyclically reduced piling, the $a_{i}$-stack starts with a letter + or - , then one can perform a cycling of the bottom tile containing that bead to the top of the piling. A physical interpretation of this procedure is obtained by replacing the sticks by concentric hula hoops, and cycling the tile around the hula hoops (see Figure 6).

Proposition 2.18. There is an algorithm which takes as its input any non-split cyclically reduced piling $p$ and which outputs a pyramidal piling that is obtained from the input piling by a finite sequence of cyclings. If the piling has $\ell$ beads, then the algorithm requires $O(\ell)$ cyclings, so its computational complexity is $O(\ell)$.

Proof. The basic procedure of the algorithm is in two steps; given a cyclically reduced piling $p$, first determine the 0 -factor $p_{0}$ of the canonical decomposition (by the method of Lemma 2.16). Secondly, cycle all the tiles belonging to $p_{0}$ in order to obtain a new piling. This procedure takes time $O(\ell)$. The algorithm is simply to iterate this basic procedure until the factor $p_{0}$ is empty. It remains to prove that there is a bound on the number of iterations which depends only on the group $A$, not on the piling $p$. In fact, if we denote $i$ the smallest index such that $p$ contains an $a_{i}$-tile, and $\Delta(p)$ the full subgraph of the defining graph $\Gamma$ defined above, we claim that $\max _{a_{j} \in \Delta(p)}$ dist $_{\Delta}\left(a_{i}, a_{j}\right)$ is an upper bound on the number of iterations, where each edge of $\Delta(p)$ has length 1 . This quantity is finite, because $\Delta(p)$ is connected, and 

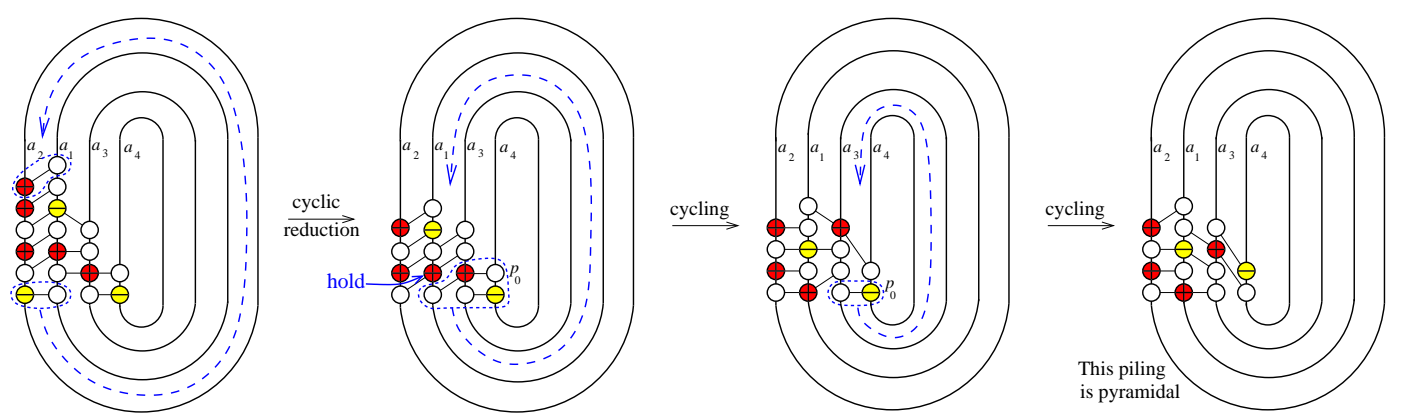

FIGURE 6. The calculation of a pyramidal piling

is bounded above by $N$, the number of generators of the group $A$ (which does not depend on the piling $p$ ). This fact is obvious from the geometrical representation, and the proof is a straightforward induction: after the first iteration of the basic procedure, no $a_{j}^{ \pm}$-beads such that $a_{j}$ is at distance 1 from $a_{i}$ in $\Delta(p)$ appear in the 0-factor; after a second iteration no $a_{j}^{ \pm}$beads such that $a_{j}$ is at distance at most 2 from $a_{i}$ in $\Delta(p)$ appear in the 0 -factor, and so on.

Now, if $w$ is a non-split reduced word, we can apply the algorithm of Proposition 2.18 to the piling $\pi(w)$ to obtain a pyramidal piling $p$. Then the words $\sigma^{\star}(p)$ and $w$ represent conjugate elements.

Definition 2.19. Let $w$ be a word in $\left\{a_{1}^{ \pm 1}, \ldots, a_{n}^{ \pm 1}\right\}^{*}$ that is reduced and cyclically reduced. We say that the word $w$ is a cyclic normal form if it is normal and all its cyclically conjugate words are normal.

Intuitively, if we regard $w$ as a cyclic word, and we start reading anywhere in the word, then the first letter that we read must always be the largest-index letter that can be extracted on the left. For instance, with the notation of Example 2.4, the word $a_{4}^{-1} a_{3} a_{2}^{-1} a_{1} a_{2} a_{1}^{-1} a_{2} a_{2}$ is not a cyclic normal form: starting from the last letter and reading cyclically, we read out $a_{2} a_{4}^{-1} \ldots$, which is already illegal, because the letters $a_{2}$ and $a_{4}$ commute, and $a_{4}^{-1}$ has a larger index than $a_{2}$, so $a_{4}^{-1}$ should come first. Another example: the word $a_{1} a_{2} a_{1}^{-1} a_{3} a_{4}^{-1} a_{2}$ is a cyclic normal form. Our linear-time solution to the conjugacy problem is based on the two following results.

Proposition 2.20. If $p$ is a non-split cyclically reduced pyramidal piling, then $\sigma^{\star}(p)$ is a cyclic normal form.

Proposition 2.21. Two cyclic normal forms represent conjugate elements if and only if they are equal up to a cyclic permutation.

Proof of Proposition 2.20. Firstly, we remark that a consequence of Lemma 2.1 is the following fact: if $a^{\epsilon}, b^{\eta}$ are letters $(\epsilon, \eta= \pm 1)$ and $w$ is a reduced word such that $b^{-\eta} w$ and $w a^{\epsilon}$ are both reduced (i.e. no word equivalent to $w$ starts and finishes with $b^{\eta}$ and $a^{-\epsilon}$, respectively) but the word $b^{-\eta} w a^{\epsilon}$ is not reduced (i.e. $w a^{\epsilon}$ is equivalent to some word that starts with $b^{\eta}$ ), then $a^{\epsilon}=b^{\eta}$ and all the letters of $w$ commute with $a$. Now, we know that $\sigma^{\star}(p)$ is a normal cyclically reduced word. For a cyclically reduced word $w$, the word $w w$ is cyclically reduced 
(this follows directly from the above fact, or from the piling representation), and all the words cyclically conjugate to the former are subwords of the latter. Therefore, in order to prove the result, it is enough to prove that the word $\sigma^{\star}(p) \sigma^{\star}(p)$ is normal. Assume that this is not the case. Since $\sigma^{\star}(p)$ is normal, we can then write $\sigma^{\star}(p)=w_{1} a_{j}^{\eta} w_{2}=v_{1} a_{i}^{\epsilon} v_{2}$ such that $a_{j}^{\eta} w_{2} v_{1}$ is initially normal but $a_{j}^{\eta} w_{2} v_{1} a^{\epsilon}$ is not. In particular, there exists $a_{k}^{\nu}$, with $k>j$, such that $a_{k}^{-\nu} a_{j}^{\eta} w_{2} v_{1}$ is reduced but $a_{k}^{-\nu} a_{j}^{\eta} w_{2} v_{1} a_{i}^{\epsilon}$ is not. Since $a_{j}^{\eta} w_{2} v_{1} a_{i}^{\epsilon}$ is a subword of $\sigma^{\star}(p) \sigma^{\star}(p)$, it is reduced. Using the above fact, we get that $a_{i}^{\epsilon}=a_{k}^{\nu}$, and $a_{k}^{\nu}$ commute with all the letters of $a_{j}^{\eta} w_{2} v_{1}$. In particular, the word $\sigma^{\star}(p)$ is equivalent to $a_{k}^{\nu} v_{1} v_{2}$. This is impossible because $k$ is greater than $j$, and $p$ is pyramidal. Therefore, $\sigma^{\star}(p) \sigma^{\star}(p)$ is normal.

Proof of Proposition 2.21. The "if" implication is obvious, we have to prove the "only if" part.

Let $w$ and $w^{\prime}$ be two cyclic normal forms that represent conjugate elements. Let $i$ be the smallest index that appears in $w$ and choose a distinguished letter $a_{i}^{\varepsilon}$ in $w$. As the words $w$ and $w^{\prime}$ are cyclically reduced, there exists a sequence of words $w_{0}=w \rightarrow w_{1} \rightarrow \cdots w_{r}=w^{\prime}$ that transforms $w$ into $w^{\prime}$, such that $w_{i+1}$ is obtained from $w_{i}$ by a commutation or a cycling transformation.

We can keep track of the distinguished letter $a_{i}^{\varepsilon}$ along the transformations: write $w_{j}=$ $w_{j}^{\prime} a_{i}^{\varepsilon} w_{j}^{\prime \prime}$. Assume the number $\ell$ of commutations that involve the distinguished letter is positive. Since $w$ is a normal word, the first commutation $w_{j} \rightarrow w_{j+1}$ that involves $a_{i}^{\varepsilon}$ is "from left to right", i.e. it is of the following form: $w_{j}=w_{j+1}^{\prime} a_{i^{\prime}}^{\varepsilon^{\prime}} a_{i}^{\varepsilon} w_{j}^{\prime \prime}$ and $w_{j+1}=w_{j+1}^{\prime} a_{i}^{\varepsilon} a_{i^{\prime}}^{\varepsilon^{\prime}} w_{j}^{\prime \prime}$ with $i^{\prime}>i$.

Now, consider the last operation $w_{p} \rightarrow w_{p+1}$ such that a letter $a_{k}^{\eta}$ is exchanged with the distinguished letter $a_{i}^{\varepsilon}$ from left to right: we have $w_{p}=w_{p+1}^{\prime} a_{k}^{\eta} a_{i}^{\varepsilon} w_{p}^{\prime \prime}$ and $w_{p+1}=w_{p+1}^{\prime} a_{i}^{\varepsilon} a_{k}^{\eta} w_{p}^{\prime \prime}$. We can also keep track of the distinguished letter $a_{k}^{\eta}$. As long as the two letters do not cross each other again in the opposite direction, we have $w_{q}^{\prime \prime} w_{q}^{\prime}=y_{q} a_{k}^{\eta} z_{q}$ such that all the letters of $y_{q}$ commute with $a_{k}$ (where $q$ satisfies $q>p$ ). In particular, $a_{i}^{\varepsilon} w_{q}^{\prime \prime} w_{q}^{\prime}$ is not initially normal. But $w^{\prime}$ is normal, so the two distinguished letters have to cross each other again in the opposite direction: there exists $s$, with $p<s<r$, such that $w_{s}=w_{s}^{\prime} a_{i}^{\varepsilon} a_{k}^{\eta} w_{s+1}^{\prime \prime}$ and $w_{s+1}=w_{s}^{\prime} a_{k}^{\eta} a_{i}^{\varepsilon} w_{s+1}^{\prime \prime}$. Hence, we have a sequence

$$
w_{p}=w_{p+1}^{\prime} a_{k}^{\eta} a_{i}^{\varepsilon} w_{p}^{\prime \prime} \rightarrow v_{p+1}^{\prime} a_{k}^{\eta} a_{i}^{\varepsilon} v_{p+1}^{\prime \prime} \rightarrow \cdots \rightarrow v_{s-1}^{\prime} a_{k}^{\eta} a_{i}^{\varepsilon} v_{s-1}^{\prime \prime} \rightarrow v_{s}^{\prime} a_{k}^{\eta} a_{i}^{\varepsilon} v_{s}^{\prime \prime} \rightarrow w_{s+1}
$$

such that each word $v_{q}^{\prime \prime} v_{q}^{\prime}$ is equal to the word $y_{q} z_{q} w_{q}^{\prime}$. Thus we obtain a new sequence from $w$ to $w^{\prime}$ with only $\ell-2$ commutations that involve the distinguished letter $a_{i}^{\varepsilon}$.

It follows that we can assume that no commutation involves the distinguished letter $a_{i}^{\varepsilon}$ along the sequence $w_{0}=w \rightarrow w_{1} \rightarrow \cdots w_{r}=w^{\prime}$. But this implies that the words $a_{i}^{\varepsilon} w_{1}^{\prime \prime} w_{1}^{\prime}$ and $a_{i}^{\varepsilon} w_{r}^{\prime \prime} w_{r}^{\prime}$ are equivalent. As they are both cyclic normal forms, they are normal words. Therefore they are equal by Proposition 2.6. Hence, the words $w$ and $w^{\prime}$ are equal up to a cyclic permutation.

Theorem 2.22. The conjugacy problem in a right-angled Artin group $A$ is linear-time on the sum of the lengths of the two input words.

Proof. Here is a summary of the algorithm: given any two words $w$ and $v$,

(i) produce the piling $\pi^{\star}(w)$, and then by cyclic reduction a cyclically reduced piling $p$; similarly for the word $v$ produce first the piling $\pi^{\star}(v)$, and cyclically reduce it to a piling $q$; 
(ii) factorize each of the pilings $p$ and $q$ into non-split factors. If the collection of subgraphs $\Delta_{i}(p)$ and $\Delta_{i}(q)$ of the defining graph $\Gamma_{A}$ do not coincide, output "NO, $w$ and $v$ do not represent conjugate elements" and stop. Otherwise,

(iii) if $p=p^{(1)} \cdot \ldots \cdot p^{(k)}$ and $q=q^{(1)} \cdot \ldots \cdot q^{(k)}$ are the factorizations found in step (ii), then for $i=1, \ldots, k$ do the following

(a) transform the non-split cyclically reduced pilings $p^{(i)}$ and $q^{(i)}$ into pyramidal pilings $\widetilde{p}^{(i)}$ and $\widetilde{q}^{(i)}$, using a sequence of cyclings. Then produce the words in cyclic normal form $\sigma^{\star}\left(p^{(i)}\right)$ and $\sigma^{\star}\left(q^{(i)}\right)$;

(b) decide whether the words in cylic normal form found in the previous steps are the same up to cyclic permutation (in linear-time, using a standard algorithm). If they are not, answer "NO" and stop.

(iv) answer "YES".

\subsection{Calculating the centralizer of an element}

The centralizer of a cyclically reduced element of $A$ has a canonical finite generating set: suppose that $w$ is a cyclically reduced word, written as a product of cyclically reduced nonsplit words $w=w_{1} \cdots w_{k}$, c.f. Section 2.2.2. Then, according to [6], for each $i$ in $\{1, \ldots, k\}$ there exists a unique maximal infinite-cyclic subgroup of $A$ containing $\left[w_{i}\right]$, generated by some cyclically reduced element $\left[z_{i}\right]$, and by $[\mathbf{2 7}]$ the centralizer of $[w]$ in $A$ is generated by

(1) the elements $\left[z_{i}\right]$, and

(2) the generators of $A$ which commute with all the generators occurring in $w$.

In the next section we will need to algorithmically determine explicit representatives of these generators, in the special case where the words $w_{i}$ are cyclic normal forms.

Proposition 2.23. There is a linear-time algorithm which takes as its input a cyclically reduced word $w$, decomposed as a product of words in cyclic normal form $w=w_{1} \cdots w_{k}$, and which outputs the canonical generating set of the centralizer of $w$.

Proof. It takes linear time to determine the graph $\Delta(w)$, and then constant time to deduce from this the generators of type (2).

Now we turn to the generators of type (1), i.e. the minimal roots $\left[z_{i}\right]$ of the elements $\left[w_{i}\right]$. As a first step, we claim that periodicity of elements is visible in their cyclic normal form. More precisely, if one of the words $w_{i}$ is equivalent to a word of the form $\widetilde{z}_{i}^{r}$ for some word $\widetilde{z}_{i}$ and some integer $r$, then the word $w_{i}$ itself is of the form $z_{i}^{r}$, for some word $z_{i}$. In order to prove this claim, we observe that $\widetilde{z}_{i}$ is equivalent to a word $z_{i}$ in cyclic normal form (because the 0 -factor of $p\left(\widetilde{z}_{i}\right)$ must divide the 0 -factor of $p\left(w_{i}\right)$, which is the trivial word). Now the word $z_{i}^{r}$ is still in cyclic normal form (c.f. the proof of Proposition 2.20), and it is equivalent to $w_{i}$. Therefore we have $z_{i}^{r}=w_{i}$.

We claim that for each of the factors $w_{i}$, the desired minimal root $z_{i}$ of $w_{i}$ is detectable in linear-time: we can calculate a pair $\left(z_{i}, r\right)$, where $z_{i}$ is a word and $r$ an integer with $z_{i}^{r}=w_{i}$, and $r$ is maximal among all such pairs. Indeed, this algorithm works as follows: consider the word $w_{i}^{*}$ obtained by removing the first letter from the word $w_{i} w_{i}$. Then find the starting point of the first occurrence of $w_{i}$ as a subword of $w_{i}^{*}$ - this can be done by standard algorithms, like the Boyer-Moore algorithm, in time $O\left(\ell_{i}\right)$, where $\ell_{i}$ denotes the length of $w_{i}$. If this starting 
point is at the $\ell_{i}$ th letter of $w_{*}$, then there is no periodicity. If on the other hand the starting point is at the $t$ th letter with $t<\ell_{i}$, then let $z_{i}$ be the prefix of $w_{i}$ of length $t$. By construction we have an equality of words $z_{i} w_{i}=w_{i} z_{i}$. This implies that the words $w_{i}$ and $z_{i}$ have a common root. By the choice of $z_{i}$, this root has to be $z_{i}$ itself and for $r:=\ell_{i} / t$ we have an equality of words $w_{i}=z_{i}^{r}$. Finally, by the choice of $t$, no prefix of $w_{i}$ of length less than $t$ can be a root of $w_{i}$, so $z_{i}$ is indeed the minimal root.

\section{The conjugacy problem in subgroups of RAAGs}

In the previous section we saw that the conjugacy problem in a fixed right-angled Artin group can be solved in linear-time on a RAM-machine with constant that depends only on the group. In this section we shall prove analogue results for a large class of subgroups of right-angled Artin groups, namely those considered in the papers $[\mathbf{1 1}, \mathbf{1 2}]$, as well as in $[\mathbf{1 9}]$.

\subsection{A class of subgroups of RAAGs}

Every right-angled Artin group $A$ admits a finite $K(A, 1)$, called the Salvetti complex of $A$, which we shall denote $Y$ and which can be constructed explicitely from the presentation of $A$. It is a cubed complex which has one single vertex, and one edge of length 1 for every generator of $A$. Moreover, for every $n$-tuple of mutually commuting generators of $A$, there is one $(n+1)$ torus in $Y$. We equip every cell, of any dimension, of this complex with the flat metric, in the sense that in the universal cover $\widetilde{Y}$ every cell is a Euclidean cube of side length 1 . Then the complex is locally $\operatorname{CAT}(0)$, and its universal cover $\widetilde{Y}$ is $\operatorname{CAT}(0)$. For instance, for the group $A=\mathbb{Z}^{2}=\left\langle a_{1}, a_{2} \mid\left[a_{1}, a_{2}\right]=1\right\rangle$, the complex $Y$ is a torus, constructed out of one vertex, two edges, and one square which glued to the 1-skeleton according to the commutation relation. See [11] for details. Notice that as soon as an orientation is chosen on each edge (i.e. simple loop) of $Y$, one obtains an explicit isomorphism between $A$ and $\pi_{1}(Y)$ such that the image of each generator $a_{i}$ of $A$ is represented by the simple loop labelled by $a_{i}$ traversed in the positive direction.

Now, suppose that $X$ is a finite locally $C A T(0)$ cubed complex, and consider a cubical map $\Phi: X \rightarrow Y$, sending each open cube of $X$ bijectively and locally isometrically to a cell of the same dimension in $Y$. If one of the vertices of $X$ is designated as its basepoint, then such a mapping induces a homomorphism $\Phi_{*}: \pi_{1}(X) \rightarrow \pi_{1}(Y)$. See Figure 7 for an example where $X$ and $Y$ are 1-dimensional complexes.

We need some more notation: for any vertex $x$ of $X$, we denote by $\Phi_{l k}: l k(x, X) \rightarrow$ $l k(\Phi(x), Y)$ the induced map from the link of $x$ in $X$ to the link of $\Phi(x)$ in $Y$ We shall be interested in the following two properties which our map $\Phi$ may have:

- The convexity property: for any vertex $x$ of $X$, and any two vertices of $l k(\Phi(x), Y)$ which belong to the image $\Phi_{l k}(l k(x, X))$ and which are connected by an edge, the connecting edge belongs to the image $\Phi_{l k}(l k(x, X))$, as well.

- The injectivity property: the map of universal covers $\widetilde{\Phi}: \widetilde{X} \rightarrow \widetilde{Y}$ is injective. In particular, $\Phi_{*}: \pi_{1}(X) \rightarrow \pi_{1}(Y)$ is a monomorphism.

We remark that a map $\Phi$ satisfying the two hypotheses is a local isometry. Now, the subgroups of the right-angled Artin group $A \cong \pi_{1}(Y)$ for which we shall solve the conjugacy problem are the fundamental groups $\pi_{1}(X)$ of cubical complexes $X$ which admit a cubical map $\Phi: X \rightarrow Y$ with the convexity and injectivity property.

REMARK 3.1. If $X$ and $Y$ are both known to be $C A T(0)$ cube complexes then the convexity property implies the injectivity property - cf. [11], Theorem 1 and the remark following. 
Conversely, the two conditions, together with the knowledge that $Y$ is $C A T(0)$, imply that the complex $X$ is itself $C A T(0)$.

The reader unfamiliar with the geometrical language used in stating the conditions should remember that the convexity and injectivity properties are satisfied by all the subgroups of right-angled Artin groups discussed in Theorem 1 of [11]. So some typical examples to keep in mind are those given in this paper. More generally, in order to get a mental image of the class of subgroups satisfying the two hypotheses, one can think of a subgroup whose Cayley graph sits in the Cayley graph of $A$ in a "flat" way. Moreover, as proven by Haglund and Wise ([19], Theorem 4.2), for a cubed complex $X$, the property of admitting a map $\Phi$ to a RAAG with the convexity and injectivity property can be characterized purely in terms of certain combinatorial conditions on the complex $X$ - they call such complexes special.

Notation. We fix some notation and conventions for the rest of the section:

- We fix once and for all a right-angled Artin group $A$ given by a presentation with generators $a_{1}, \ldots, a_{N}$, and we denote by $Y$ the cubed complex associated with $A$. We fix an orientation on every edge of $Y$ and identify $A$ with $\pi_{1}(Y)$, using the chosen orientations.

- We also fix a finite cubed complex $X$ and $\Phi: X \rightarrow Y$ a cubical map satisfying the convexity and injectivity condition. Finally, we fix a label $x_{1}, x_{2}, x_{3}, \ldots$ for each vertex of $X$.

We will not directly solve the conjugacy problem in the fundamental group of $X$, but a more general problem, namely the conjugacy problem in the fundamental groupoid of $X$, in linear-time. First, we explain what precisely that means.

Let us fix a (positive) orientation for each edge of the complex $X$ by pulling back along $\Phi$ the orientation of edges in $Y$. An element of the fundamental groupoid is, by definition, a homotopy class of paths (with fixed endpoints) from some vertex $x_{i}$ to some vertex $x_{j}$. Such an element of the fundamental groupoid can be represented by a finite sequence of successive directed edges, which may be traversed in the positive or in the negative direction. We shall call such a sequence an edge path from $x_{i}$ to $x_{j}$. Similarly in $Y$ we have an analogue notion of an edge path as a homotopy class of path specified as a sequence of positively or negatively directed edges.

We shall use the following very convenient way of coding edge paths in $X$ and $Y$ : in $Y$, we shall simply identify closed edge paths with words in the letters $a_{1}^{ \pm 1}, \ldots, a_{N}^{ \pm 1}$. As for $X$, the map $\Phi$ gives rise to a coding of edge paths in $X$ by based words.

Definition 3.2. A based word is a word of the form $x_{i} w x_{j}$, where $x_{i}$ and $x_{j}$ are vertices of $X$, and $w$ is the image under $\Phi$ of an edge path in $X$ starting at $x_{i}$ and ending at $x_{j}$. The vertex $x_{i}$ is called the base vertex of the based word.

In other words, the edge path $x_{i} w x_{j}$ is by definition the pullback to $X$ of the path $w$ in $Y$ which starts at $x_{i}$ and ends at $x_{j}$. Notice that not every word of the form $x_{i} w x_{j}$, with $x_{i}$ and $x_{j}$ vertices of $X$ and $w$ a word with letters in $\left\{a_{1}^{ \pm 1}, \ldots, a_{N}^{ \pm 1}\right\}$, is a based word. However, when it is, then it uniquely determines an edge path in $X$, because of the injectivity property. For instance, if $x_{i} w x_{j}$ is a based word, and if the word $w$ can be written as a concatenation $w=w_{1} w_{2}$, then there exists a unique vertex $x_{k}$ such that $x_{i} w_{1} x_{k}$ and $x_{k} w_{2} x_{j}$ are based words. For an example of based words, see again Figure 7.

Two elements of the fundamental groupoid of $X$ can be multiplied if the terminal vertex of the first coincides with the initial vertex of the second. In terms of based words, $\left(x_{i} w_{1} x_{j}\right)$. 
$\left(x_{j} w_{2} x_{k}\right)=x_{i} w_{1} w_{2} x_{k}$. Two loops in $X$ are freely homotopic if and only if they represent conjugate elements of the fundamental groupoid. If the loops are represented by based words $x_{1} w x_{1}$ and $x_{2} v x_{2}$, then this equivalent to the existence of a based word $x_{1} u x_{2}$ such that the elements of the fundamental groupoid represented by $x_{1} u v u^{-1} x_{1}$ and $x_{1} w x_{1}$ coincide.

We are now ready to state our main result. The proof will occupy the whole rest of the paper:

TheOREM 3.3. Given two based words $x_{1} w x_{1}$ and $x_{2} v x_{2}$, one can decide whether they represent freely homotopic loops in $X$. Moreover, if $w$ and $v$ have length $\ell_{1}$ and $\ell_{2}$, respectively, the decision can be performed by an algorithm which takes time $O\left(\ell_{1}+\ell_{2}\right)$ on a $R A M$ machine, where the linear constants depend on $X, Y$ and $\Phi$ only.

\subsection{Base points and homotopies in the cubical complex $X$}

Warning. Suppose $\alpha$ and $\beta$ are two closed edge paths in $X$ based at a common vertex $x$. One might think that $\alpha$ and $\beta$ represent conjugate elements of $\pi_{1}(X)$ if and only if the words $\Phi(\alpha)$ and $\Phi(\beta)$ represent conjugate elements of $A$. This, however, is wrong, and an explicit counterexample, illustrating the underlying basepoint problem, is given in Figure 7.

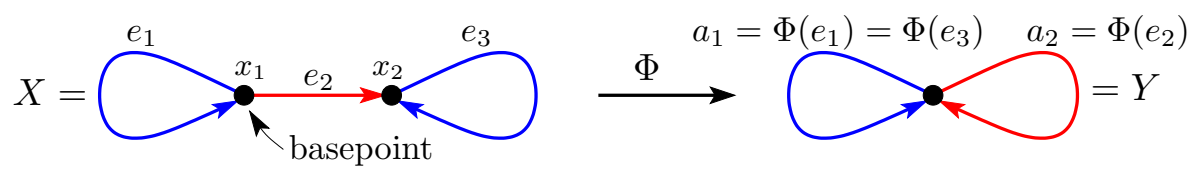

Figure 7. $\pi_{1}(Y)$ is the free group on two generators $a_{1}=\Phi\left(e_{1}\right)=\Phi\left(e_{3}\right)$ and $a_{2}=\Phi\left(e_{3}\right)$. The loops $e_{1}$ and $e_{2} e_{3} e_{2}^{-1}$ are not conjugate as elements of $\pi_{1}(X)$, whereas their images in $\pi_{1}(Y)$ are. In order to describe the loops in $X$ it is better to use the based words $x_{1} a_{1} x_{1}$ and $x_{1} a_{2} a_{1} a_{2}^{-1} x_{1}$. The latter is conjugate to $x_{2} a_{1} x_{2}$.

In order to prepare the proof of Theorem 3.3, let us study what homotopies of paths in $X$ look like.

If $\alpha$ is an edge path in $X$ giving rise to a based word $x_{i} w x_{j}$, and if $x_{i} \widetilde{w} x_{j}$ is a based word obtained from $x_{i} w x_{j}$ by one application of a commutation relation (corresponding to a homotopy of a path in $Y$ across a square) then there exists an edge path $\widetilde{\alpha}$ in $X$, starting from the same vertex as $\alpha$ and homotopic to $\alpha$, which gives rise to the based word $x_{i} \widetilde{w} x_{j}-$ this is an immediate consequence of the convexity condition. Similarly, free cancellations in $w$ can be realised by cancellations of backtracking path segments in $\alpha$.

Let us now look more generally at free homotopies of loops in $X$, i.e., homotopies that move the basepoint.

Definition 3.4. Suppose that $x w x$ is a based word. A parallel transport of $x w x$ is a replacement of the vertex $x$ by a vertex $x^{\prime}$, where $x^{\prime}$ is obtained from $x$ by walking along an oriented edge $e$ with the property that the element $\Phi(e)$ of $A$ commutes with all the generators of $A$ occurring in the word $w$.

Geometrically, this move corresponds to replacing a closed path based at $x$ by a parallel one based at $x^{\prime}$, where $x$ and $x^{\prime}$ are joined by an edge $e$. The two paths together bound an annulus-shaped region of $X$. Notice that, under $\Phi$, the two paths have the same image $w$ in $Y$. Another way of moving the basepoint of a loop is to push it along the loop: 
Definition 3.5. Suppose that $w=x y_{1} y_{2} \ldots y_{\ell} x$ is a based word, and denote by $e$ the unique edge of $X$ that has one of its extremities equal to $x$ and such that $\Phi(e)=y_{1}$. A based cycling of the based word $w$ is its replacement by the word $x^{\prime} y_{2} \ldots y_{\ell} y_{1} x^{\prime}$, such that the vertex $x^{\prime}$ is the second extremity of the edge $e$.

Geometrically, if $\alpha$ is a loop in $X$ based at a vertex $x$, and described by a based word $x y_{1} y_{2} \ldots y_{\ell} x$, and if we apply a cycling operation (in the sense of section 2) to the word $y_{1} y_{2} \ldots y_{\ell}$, then this cycling can be pulled back to $X$ to a based cycling of the based word, yielding a loop $\widetilde{\alpha}$, which looks exactly like $\alpha$, except that it based at a different vertex $x^{\prime}$, "one notch further along the loop".

EXAMPLE 3.6. In the example of Figure 7, we can apply a based cycling to the based word $x_{1} a_{2} a_{1} a_{2}^{-1} x_{1}$, yielding $x_{2} a_{1} a_{2}^{-1} a_{2} x_{2}$. After a cancellation, we obtain the based word $\gamma_{2}=$ $x_{2} a_{1} x_{2}$. We note that this is different from the based word $\gamma_{1}=x_{1} a_{1} x_{1}$, which was also discussed in that example - in fact, the based words $x_{2} a_{1} x_{2}$ and $x_{1} a_{1} x_{1}$ are not even related by parallel transport (because $\Phi\left(e_{2}\right)=a_{2}$ does not commute with $a_{1}$ ). As we shall see in Lemma 3.9, this implies that the two loops $e_{1}$ and $e_{2} e_{3} e_{2}^{-1}$ are not freely homotopic in $X$.

Also note that a cyclic reduction of a word on the generators of $A$ and their inverses can be decomposed as a cycling, followed by a usual cancellation of letters, and each of these operations can be pulled back to operations on the loop in $X$. Summarizing the last few paragraphs, we have the following

Key Observation 3.7. If $\alpha$ is a loop in $X$ then all non-length-increasing free homotopies of the loop $\Phi(\alpha)$ in $Y$ can be pulled back to free homotopies of $\alpha$. Thus for a based word $x w x$, all cancellations, applications of commutation relations, cyclings, and cyclic reductions of the word $w$ can be pulled back to analogue cancellations, commutation relations, and based cyclings of the based word. Similarly, if $x_{1} w x_{2}$ is a based word, and if the word $w$ can be transformed into a word $w^{\prime}$ by applying cancellations and commutation relations, then $x_{1} w^{\prime} x_{2}$ is again a based word.

\subsection{The linear-time solution to the conjugacy problem}

We recall that we are considering two based words $x_{1} w x_{1}$ and $x_{2} v x_{2}$ representing two loops in $X$ traversing $\ell_{1}$ and $\ell_{2}$ edges, respectively. A necessary condition for these loops being conjugate in the fundamental groupoid of $X$ is that the words $w_{1}$ and $w_{2}$ represent conjugate elements of the right-angled Artin group $A$. In geometric terms, for the two loops to be freely homotopic in $X$, their images under $\Phi$ in $Y$ must be freely homotopic. This is a condition which we can check in time $O\left(\ell_{1}+\ell_{2}\right)$ by the results of Section 2. However, this condition is not sufficient, as seen in Example 3.6. So let us now try to refine this approach.

Proposition 3.8. There is an algorithm with running time $O\left(\ell_{1}+\ell_{2}\right)$ whose input consists of two based words $x_{1} w x_{1}$ and $x_{2} v x_{2}$ of lengths $\ell_{1}$ and $\ell_{2}$, and which outputs

(i) either the information that they do not represent freely homotopic loops in $X$, or

(ii) two based words $x_{1}^{\prime} \widetilde{w}_{1} \ldots \widetilde{w}_{k} x_{1}^{\prime}$ and $x_{2}^{\prime} \widetilde{w}_{1} \ldots \widetilde{w}_{k} x_{2}^{\prime}$, representing two loops in $X$ which are respectively freely homotopic to the original two, and where the $\widetilde{w}_{i}$ are mutually commuting cyclic normal forms. 
Proof of Proposition 3.8. As seen in Section 2 we can decide in linear-time whether $w$ and $v$ represent conjugate elements of $A$. If they do not, then the two based words do not represent conjugate elements of the fundamental groupoid either, and it suffices to output this information (case (1)).

For the rest of the proof we have to deal with the case where $w$ and $v$ do represent conjugate elements of $A$.

We already know from Section 2 that the word $w$ can, by a sequence of cancellations, commutation relations and cyclings be transformed into a word $w^{\prime}$ with the required decomposition $w^{\prime}=w_{1}^{\prime} \ldots w_{k}^{\prime}$. Moreover, we know how to calculate the word $w^{\prime}$ in linear-time.

We also know from the Key Observation 3.7 above that the transformation of the word $w$ into the word $w^{\prime}$ can be pulled back to a transformation of the based word $x_{1} w x_{1}$ into a based word $x_{3} w^{\prime} x_{3}$. Our next task is to determine the corresponding base vertex $x_{3}$ in linear-time.

We shall fulfill this task by "carrying along information about the base vertex in $X$ during the algorithm". While running the algorithm of Section 2 , the only steps that affect the base vertex are the cyclings of pilings (including cyclic reductions of pilings, which can be decomposed as cyclings, followed by cancellations of tiles): when we cycle an $a_{j}^{ \pm}$-tile, we have to determine how the base vertex is affected. However, this can be done simply by a lookup in a finite, precalculated list: for every vertex $x$ of $X$, for every generator $a_{j}$ of $A$, and for every $\epsilon \in\{-1,1\}$, this list must tell us at which vertex of $X$ we arrive if we pull back the loop $a_{j}^{\epsilon} \in A=\pi_{1}(Y)$ to a path in $X$ starting at $x$ (if that is possible). Since the algorithm of Section 2 performs a linearly bounded number of cyclings, we can calculate the new base vertex $x_{3}$ in time $O\left(\ell_{1}\right)$.

In a similar manner we can algorithmically transform the based word $x_{2} v x_{2}$ into a word $x_{2}^{\prime} \widetilde{w} x_{2}^{\prime}$, where $\widetilde{w}$ is equipped with an analogue decomposition $\widetilde{w}=\widetilde{w}_{1} \ldots \widetilde{w}_{j}$.

But since $w$ and $v$ represented conjugate elements of $A$, we have, by the results of Section 2 , that the words $w^{\prime}$ and $\widetilde{w}$ are in fact the same, at least after a reordering of the factors of $w^{\prime}$ and a linearly bounded number of cyclings of each factor $w_{i}^{\prime}$; in particular, we have $j=k$. Moreover, the Boyer-Moore algorithm tells us how many letters from each factor we have to cycle in order to achieve this. Thus we can transform the based word $x_{3} w^{\prime} x_{3}$ into the based word $x_{1}^{\prime} \widetilde{w} x_{1}^{\prime}$ for some vertex $x_{1}^{\prime}$, using a reordering of the factors (which does not affect the base vertex) and a linearly bounded number of based cyclings.

Thus in order to prove Theorem 3.3, it is enough to prove it for the special case $v=w=$ $\widetilde{w}_{1} \ldots \widetilde{w}_{k}$, where the words $\widetilde{w}_{1}, \ldots, \widetilde{w}_{k}$ are mutually commuting cyclic normal forms. (For instance, this is the situation of Example 3.6, where we need to decide if the based words $x_{1} a_{1} x_{1}$ and $x_{2} a_{1} x_{2}$ represent freely homotopic loops in $X$.) For the rest of the proof of Theorem 3.3 we fix a word $\widetilde{w}$ of length $\ell$, with such a decomposition $\widetilde{w}=\widetilde{w}_{1} \ldots \widetilde{w}_{k}$, and our aim is to decide in time $O(\ell)$ whether two based words $x_{1} \widetilde{w} x_{1}$ and $x_{2} \widetilde{w} x_{2}$ represent freely homotopic loops.

Suppose a based word $x_{1} \widetilde{u} x_{2}$ is such that $x_{1} \widetilde{u} \widetilde{w} \widetilde{u}^{-1} x_{1}$ and $x_{1} \widetilde{w} x_{1}$ represent the same element of the fundamental groupoid. Then in particular the elements of $A$ represented by $\widetilde{u}$ and $\widetilde{w}$ commute: we have $[\widetilde{u}][\widetilde{w}][\widetilde{u}]^{-1}=[\widetilde{w}]$ in $A$.

As seen in Section 2.3, and using the notation of this section, the word $\widetilde{u}$ is equivalent to another word $u$ of the form

$$
u=z_{1}^{p_{1}} \ldots z_{k}^{p_{k}} \zeta
$$

where $p_{1}, \ldots, p_{k}$ are integers and $\zeta$ is a word whose letters are generators of $A$ which commute with, but are different from, all the generators occurring in $w$, and their inverses. We shall call such a word $u$ a word in preferred form. We define the norm $\|u\|$ of $u$ by

$$
\|u\|=\sum_{i=1}^{k}\left|p_{i}\right|+\operatorname{length}(\zeta)
$$


We are now ready state an algorithmically checkable criterion for $x_{1} \widetilde{w} x_{1}$ and $x_{2} \widetilde{w} x_{2}$ representing conjugate elements (i.e. representing freely homotopic loops in $X$ ):

LEMmA 3.9. The two based words $x_{1} \widetilde{w} x_{1}$ and $x_{2} \widetilde{w} x_{2}$ represent conjugate elements in the fundamental groupoid if and only if there exists a based word $x_{1} u x_{2}$ such that $u$ is a word in preferred form with

$$
\|u\| \leqslant \#\{\text { vertices of } X\}
$$

Proof. We first suppose that an edge path $x_{1} u x_{2}$ exists, where $u$ is a word in preferred form. Then the word $u \widetilde{w} u^{-1}$ can be transformed into the word $\widetilde{w}$ by a finite number of commutation relations and cancellations (but no length-increasing transformations). By Key Observation 3.7, this homotopy can be pulled back to $X$, to yield a based homotopy between the paths in $X$ represented by the based words $x_{1} u \widetilde{w} u^{-1} x_{1}$ and $x_{1} \widetilde{w} x_{1}$. In other words, the elements $x_{1} \widetilde{w} x_{1}$ and $x_{2} \widetilde{w} x_{2}$ are conjugate, with conjugating element $x_{1} u x_{2}$.

Conversely, let us suppose that a conjugating element in the fundamental groupoid exists, and is represented by a based word $x_{1} \widetilde{u} x_{2}$. This means that there exists an edge path in $X$ from $x_{1}$ to $x_{2}$ such that reading out the edge labels along the path yields the word $\widetilde{u}$. As seen before, $[\widetilde{u}]$ belongs to the subgroup of $A$ generated the elements $\left[z_{1}\right], \ldots,\left[z_{k}\right]$ and $\left[a_{j_{1}}\right], \ldots,\left[a_{j_{m}}\right]$. Thus there is a word $u$ in preferred form which can be obtained from $\widetilde{u}$ by a sequence of reductions and commutation relations. By Key Observation 3.7, $x_{1} u x_{2}$ is also a based word, i.e. it also represents an edge path in $X$.

We have shown the existence of a based word $x_{1} u x_{2}$ with $u$ a word in preferred form, and without loss of generality we can suppose that $u$ is chosen so that $\|u\|$ is minimal among all such based words.

Now for $t$ in $\{0, \ldots,\|u\|\}$ let us denote by $x(t)$ the vertex of $X$ obtained by a walk in $X$ starting at $x_{1}$ and following the edges of $X$ according to the $t$ first subwords. Now, if this function

$$
\{0,1, \ldots,\|u\|\} \longrightarrow\{\text { vertices of } X\}, \quad t \mapsto x(t)
$$

is not injective (for instance, if $\|u\|$ is larger than the number of vertices of $X$ ), then there exists a strictly shorter edge path in $X$ represented by a based word $x_{1} u^{\prime} x_{2}$ with $u^{\prime}$ also in preferred form, obtained by cutting out some segment of the previous edge path (c.f. the paragraph following Definition 3.2). This is in contradiction to the choice of $u$, and we can conclude that we have $\|u\| \leqslant \#\{$ vertices of $X\}$.

Let us now prove that the condition of Lemma 3.9 can be checked algorithmically in lineartime, i.e. in time $O(\ell)$, where $\ell$ is the length of the word $w$.

Firstly, recalling that the centralizer of $[\widetilde{w}]$ is generated by a finite number of elements (some of them represented by the words $z_{1}, \ldots, z_{k}$ and the others equal to certain generators of $A$ ), we observe that there is a universal upper bound on the number of generators, namely the number of generators of $A$. Moreover, as seen in Proposition 2.23, words representing these generators can be determined in linear time.

Now there is a very simple-minded linear-time algorithm to check for the existence of a conjugating element: for all words $u$ in preferred form satisfying condition (3.1) check whether $x_{1} u x_{2}$ is a based word, i.e. whether there exists an edge path in $X$ represented by the based word $x_{1} u x_{2}$. Indeed, there is a universal bound on the number of words to be checked, and for each word $u$ the check takes linear time (since the length of the words $z_{i}$ can grow linearly with the length of $\widetilde{w}$ ). 
Here is a summary of the whole algorithm: given two based words $x_{*} w x_{*}$ and $x_{*} v x_{*}$ representing loops $\alpha$ and $\beta$ in $X$,

(1) Apply steps (i) and (ii) of the algorithm of Section 2.2, always carrying along the base vertex, to find graphs $\Delta_{j}(w)(j=1, \ldots, k), \Delta_{j}(v)\left(j=1, \ldots, k^{\prime}\right)$, base vertices $x_{1}, x_{2}$, and based words $x_{1} w_{1} \ldots w_{k} x_{1}$ and $x_{2} v_{1} \ldots v_{k^{\prime}} x_{2}$ representing loops that are freely homotopic to $\alpha$ and $\beta$.

(2) If $k \neq k^{\prime}$, or if the collections of full subgraphs $\Delta_{j}(w)$ and $\Delta_{j}(v) \subset \Gamma$ are not the same, or if for some $j$ between 1 and $k$ the words $v_{j}$ and $w_{j}$ do not have the same length $\ell_{j}$, return "NO".

(3) Apply step (iii) (a) of the algorithm of Section 2.2 to each of the $k$ factors, always carrying along the base vertices, to transform $x_{1} w_{1} \ldots w_{k} x_{1}$ into a based word $x_{3} w_{1}^{\prime} \ldots w_{k}^{\prime} x_{3}$ and similarly $x_{2} v_{1} \ldots v_{k} x_{2}$ into $x_{2}^{\prime} \widetilde{w}_{1} \ldots \widetilde{w}_{k} x_{2}^{\prime}$, where all words $w_{i}^{\prime}$ and $\widetilde{w}_{i}$ are cyclic normal forms.

(4) For each factor, use a standard pattern matching algorithm to decide if $w_{i}^{\prime}=\widetilde{w}_{i}$ as cyclic words. If no, return "NO". If yes, keep in mind how many cyclings of each factor $w_{i}^{\prime}$ are required to achieve equality $w_{i}^{\prime}=\widetilde{w}_{i}$ as (non-cyclic) words.

(5) Perform the required based cyclings of $x_{3} w_{1}^{\prime} \ldots w_{k}^{\prime} x_{3}$ to obtain a based word of the form $x_{1}^{\prime} \widetilde{w}_{1} \ldots \widetilde{w}_{k} x_{1}^{\prime}$.

(6) Calculate the minimal roots $z_{i}$ of the words $\widetilde{w}_{i}$, as explained in Section 2.3. Also determine the set of generators that commute with all the letters occurring in the words $\widetilde{w}_{i}$, but do not occur in any of them.

(7) Check, for all words $u$ in preferred from satisfying condition (3.1), whether there exists an edge path in $X$ represented by the based word $x_{1}^{\prime} u x_{2}^{\prime}$. If for one of the words $u$ the answer is affirmative, then return "YES". Otherwise return "NO".

Questions 3.10. Can our techniques be used to say anything about the conjugacy problem in Coxeter- or Artin groups? Does the fundamental group of any compact, locally CAT $(0)$ cubical complex, even a non-special one, have a linear-time solution to the conjugacy problem (c.f. $[25])$ ?

Acknowledgements. We thank Sam Sang-Hyun Kim, Tim Hsu, Lucas Sabalka, and Michah Sageev for interesting conversations.

\section{References}

1. A Abrams, Configuration spaces of colored graphs, Geometriae Dedicata 92 (2002) 185-194.

2. A Abrams and R Ghrist, Finding topology in a factory: configuration spaces, Amer. Math. Monthly 109(2) (2002) 140-150.

3. A Abrams and R Ghrist, State Complexes for Metamorphic Robots, The International Journal of Robotics Research 23 (7-8) (2004) 809-824.

4. A V Aho, J E Hopcroft, J D Ullman, The design and analysis of computer algorithms, Addison-Wesley (1974).

5. A Baudisch, Kommutationsgleichungen in semifreien Gruppen, Acta Math. Acad. Sci. Hungar. 29 (1977) $235-249$.

6. A Baudisch, Subgroups of semifree groups, Acta Math. Acad. Sci. Hungar. 38 (1981) 19-28.

7. R S Boyer and J S Moore, A fast string searching algorithm, Comm. of the ACM 20 (10) (1977) $762-772$.

8. M R BRIDSON and A HAEFLIGER, Metric spaces of non-positive curvature, Springer Grundlehren Series, Vol. 319 (1999).

9. P CARTiER and D FoATA, Problèmes combinatoires de commutation et réarrangements, Lecture Notes in Math. 85, Springer-Verlag (1969).

10. R Charney, An introduction to right-angled Artin groups, Geometriae Dedicata 125 (2007) $141-158$.

11. J CRISP and B WIEST, Embeddings of graph braid and surface groups in right-angled Artin groups and braid groups, Algebr. Geom. Toplogy 4 (2004) 439-472. 
12. J CRISP and B WiEst, Quasi-isometrically embedded subgroups of braid and diffeomorphism groups, Trans. A.M.S. 359 (2007) 5485-5503.

13. C Duboc, Commutation dans les monoïdes libres, un cadre théorique pour l'étude du parallélisme, Thèse de doctorat, Paris (1986).

14. D B A Epstein and D Holt, The linearity of the conjugacy problem in word-hyperbolic groups, Internat. J. Algebra Comput. 16 (2006), no. 2, 287-305.

15. D FARley and L Sabalka, Discrete Morse theory and graph braid groups, Alg. and Geom. Topology 5 (2005) 1075-1109.

16. D FARLEY and L SABALKA, On the cohomology rings of tree braid groups, preprint arXiv:math.GR/0602444.

17. $\mathrm{R}$ Ghrist and V Peterson, The geometry and topology of reconfiguration, Advances in Applied Mathematics 38 (2007) 302-323.

18. D Gusfield, Algorithms on Strings, Trees, and Sequences: Computer Science and Computational Biology, Cambridge University press (1997).

19. F Haglund and D Wise, Special cube complexes, Geom. Funct. Anal. 17 (2008), no. 5, 1551-1620.

20. F HAgLund and D Wise, Coxeter groups are special, preprint (2008).

21. D E Knuth, J H Morris and V R PRATt, Fast pattern-matching algorithms, SIAM J Computing 6 (2) (1977) 323-350.

22. D Krob, J Mairesse, and I Michos, Computing the average parallelism in trace monoids, Discrete Math. 273 (2003) 131-162.

23. F Lalonde, Contribution à l'étude des empilements, Doctoral thesis, Montréal (1990).

24. H-N Liu, C Wrathall, K Zeger, Efficient solution of some problems in free partially commutative monoids, Information and Computation 89 (1990) 180-198.

25. G Niblo and L ReEves, The geometry of cube complexes and the complexity of their fundamental groups, Topology 37 (1998) 621-633.

26. L SabalKa, Embedding right-angled Artin groups into graph braid groups, Geom. Dedicata 124 (2007), 191-198.

27. H Servatius, Automorphisms of Graph Groups, J. Algebra 126 (1987) 34-60.

28. G Steven, String searching algorithms, World Scientific (1994).

29. L VAN WyK, Graph groups are biautomatic, J. Pure Appl. Algebra 94 (1994) 341-352.

30. X Viennot, Algèbres de Lie libre et monoïdes libres, Lecture Notes in Math. 691, Springer-Verlag (1978).

31. C Wrathall, Free partially commutative groups, in "Combinatorics, computing and complexity" (Ed. $D-Z \mathrm{Du}$ and $\mathrm{G} \mathrm{Hu}$ ) 195-216, Kluwer academic/Science press, Norwell, MA (1989).

Institut de Mathémathiques de Bourgogne, CNRS UMR 5584

Université de Bourgogne, B.P. 47870, 21078 Dijon cedex, France

jcrisp@gmail.com

IRMAR, CNRS UMR 6625, Campus de Beaulieu

Université de Rennes 1, 35042 Rennes, France

bertold.wiest@univ-rennes1.fr
Laboratoire de Mathématiques Nicolas Oresme, CNRS UMR 6139

Université de Caen, 14032 Caen cedex, France

eddy.godelle@math.unicaen.fr 